\title{
Fractional-order Control for Robust Position/Yaw Tracking of Quadrotors with Experiments
}

\author{
C. Izaguirre-Espinosa ${ }^{1}$, A.J. Muñoz-Vázquez ${ }^{2 *}$, A. Sánchez-Orta ${ }^{3}$, V. Parra-Vega ${ }^{3}$, I. Fantoni ${ }^{4,5}$
}

\begin{abstract}
Quadrotors are highly maneuverable light weight drones, which are prone to aerodynamic disturbances, vibrations and uncertainties. These factors stand for a problem that demands robust control laws. For position tracking, the control problem is exacerbated because the plant is underactuated in the coordinates of interest, requiring a high performance attitude tracking to resolve underactuation. In this paper, a novel fractional-order controller is proposed by considering a well-posed map that relates the position/yaw control to desired attitude references. The attitude control is continuous and enforces and sustains a sliding motion in finite-time for exponential convergence of the tracking errors to fulfill a "virtual" position controller. The resulting closed-loop system is robust against continuous disturbances that are not necessarily differentiable in the conventional sense. A numerical study based on simulations is presented to analyze the advantages of the fractional actions to design a physically realizable controller, and experiments are discussed to expose the reliability of the proposed fractional scheme implemented in an ' $X$ ' configuration quadrotor.
\end{abstract}

Index Terms-Unmanned Aerial Vehicles; Robust Attitude Control; Position/Yaw Tracking; Fractional-order Control; Disturbance Rejection

\section{INTRODUCTION}

$\mathbf{Q}$ UADROTORS have attracted the worldwide attention since their vertical take off and landing (VTOL) capabilities outperform other robotic unmanned drones. However, to obtain the expected aerodynamic advantages of quadrotors, the full dynamic model must be studied, which, unfortunately, is highly nonlinear, coupled and underactuated, furthermore, its lightweight structure is prone to aerodynamic disturbances, [1], [2]. The control design of quadrotors has mainly been addressed neglecting the full dynamic model, due to inherent difficulties to deal with the underactuated nonlinear model, and in particular for zero yaw. In [3], an output feedback scheme is proposed for semiglobal stability based on backstepping algorithm, and [4] reports backstepping sliding modes. A geometric control based on $\mathrm{SE}(3)$ is presented in [5] proposing a hierarchical control law for the position stabilization of a VTOL. Nonetheless, these schemes assume yaw zeroing and

1. Monterrey Institute of Technology and Higher Education (ITESM), Campus Sonora Norte, Hermosillo Sonora, Mexico. Email: carlos.iza.es@itesm.mx

2. Mechatronic Engineering, Polytechnic University of Victoria (UPV), Ciudad Victoria Tamaulipas, Mexico. Email: amunozv@upv.edu.mx

3. Robotics and Advanced Manufacturing, Center for Research and Advanced Studies (CINVESTAV), Saltillo Coahuila, México. Email: (anand.sanchez, vparra) @cinvestav.mx

4. Sorbonne Universités, Université de Technologie de Compiègne, CNRS, UMR 7253 Heudiasyc, 60200 Compiègne, France.

5. LS2N UMR CNRS 6004, 1 rue de la Noë, BP 92101, 44321 Nantes Cedex 3, France. Email: isabelle.fantoni@ls2n.fr

*Corresponding author depend on the exact model, either assuming knowledge of the regressor, and/or plant parameters to build some sort of feedback linearization, [6]. In addition, the robust position tracking is proposed in [7] using well-posed attitude representation based on unit quaternions to avoid singularities of the Euler representation [8], [9]. The robust position tracking, including time-varying yaw, when the dynamic model is unknown and subject to non-differentiable disturbances, is yet an open problem for singularity free kinematics. Also, fractional control schemes have been proposed for quadrotor control in [21], [22], based on sliding modes, but the problem of not necessarily differentiability disturbance rejection by means of a continuous attitude controller to enforce exponentially a virtual position controller has been overlooked.

The contributions of this paper can be enlisted as follows:

- A novel fractional-order controller for quadrotors to guarantee robust exponential tracking of attitude dynamics.

- A continuous control signal to alleviate chattering while guaranteeing robustness against continuous but nondifferentiable disturbances.

- Underactuation is solved based on a virtual position control concept.

- Experimental results are presented.

Next Section presents the dynamical model as well as the control problem. Section III presents fundamentals on Fractional Calculus. Sections IV and V show the control design, and Sections VI and VII present simulation and experimental results. Finally, conclusions are given in Section VIII.

\section{The Dynamic Model}

The quadrotor dynamic model is represented by three coupled nonlinear subsystems: the position dynamics, the kinodynamic constraint and the attitude dynamics. These subsystems describe the motion of a rigid body evolving in a threedimensional space subject to one main thrust $\mathcal{T}$, and three moments with respect to the center of mass $(\mathrm{CoM})$, [10], provided by each thrust force $f_{i}$ of each propeller, see Fig. 1. Let $\mathcal{I}=\left\{e_{1}, e_{2}, e_{3}\right\}$ and $\mathcal{A}=\left\{e_{x}^{b}, e_{y}^{b}, e_{z}^{b}\right\}$ be the earth fixed (inertial) and body fixed frames, whose origin coincides with the $C o M$. The orientation of the rigid body is described by an orthogonal rotation matrix $\mathcal{R} \in S O(3): \mathcal{A} \rightarrow \mathcal{I}$, which can be parameterized by Euler angles $(\phi, \theta, \psi)^{T} \in \mathcal{I}$, roll, pitch and yaw, respectively. The dimension of the actuation space is 4 for the 6 dimensional quadrotor, thus, the main concerns is fourfold: $i$ ) to resolve underactuation in order to control the translational coordinates $(x, y)$, ii) to reject attitude/position aggressive disturbances, iii) to establish a 


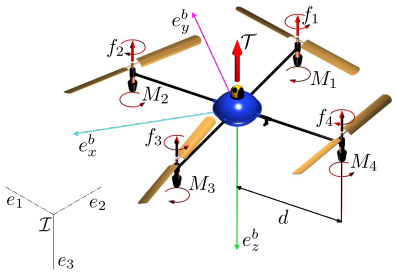

Fig. 1. Motors $M_{i}$ provide the main thrust $\mathcal{T}$ and moments by controlling the thrust force of each motor, $f_{i}=k_{i} w_{p_{i}}^{2}$, for $k_{i}$ an aerodynamical constant, and $w_{p_{i}}$ the angular velocity of the $i$-th propeller.

well-posed attitude kinematic representation, and $i v$ ) to provide a continuous controller to avoid high frequencies that may deplete the limited onboard batteries or be too complicated for the rotor drivers to handle.

\section{A. Position Dynamics}

The Newton's second law gives rise to the position dynamics of the quadrotor as

$$
m \ddot{\xi}=-\mathcal{T} \mathcal{R} e_{z}+m g e_{z}+d_{p}(t)
$$

where $\xi=(x, y, z)^{T} \in \mathcal{I}$ denotes the position of the CoM, $m$ is the mass of the quadrotor, $e_{z}=(0,0,1)^{T}, g \in \mathbb{R}$ represents the the gravitational acceleration, and $d_{p}(t) \in \mathcal{I}$ stands for external unknown position disturbances.

The $3 D$ position dynamics has only 1 control input given by the scalar thrust $\mathcal{T}=\sum_{1}^{4} f_{i}$, for $f_{i}=k w_{p_{i}}^{2}$ the vertical force provided by each propeller rotating at angular velocity $w_{p_{i}}$, which is located at a symmetrical distance $d$ wrt the CoM, see Fig. 1. On actuation space, notice that $\mathcal{T} \mathcal{R} e_{z}=$ $\mathcal{T}\left(r_{13}, r_{23}, r_{33}\right)^{T} \in \mathbb{R}^{3}$ but $\mathcal{T} \in \mathbb{R}$, thus $\mathcal{T} r_{13}, \mathcal{T} r_{23}$ and $\mathcal{T} r_{33}$ can be declared as virtual inputs to control position, which requires a scheme to resolve underactuation.

\section{B. Attitude Dynamics}

The Newton-Euler formulation yields

$$
\begin{aligned}
\dot{\mathcal{R}} & =\mathcal{R} \omega^{\times}, \\
\mathbf{J} \dot{\omega} & =-\omega^{\times} \mathbf{J} \omega+\tau+d_{a}(t)
\end{aligned}
$$

where $\omega=\left(\omega_{1}, \omega_{2}, \omega_{3}\right)^{T} \in \mathcal{A}$ denotes the angular velocity, $\omega^{\times}$is the skew-symmetric of the vector $\omega, \mathbf{J} \in \mathbb{R}^{3 \times 3}$ models the inertia wrt the $C o M$ in $\mathcal{A}, \tau \in \mathcal{A}$ represents three independent control inputs, and $d_{a}(t) \in \mathcal{A}$ stands for the attitude disturbance. There are 3 independent controllers for the attitude dynamics (3), which are given by the moments $\tau=\left(M_{\phi}, \quad M_{\theta}, M_{\psi}\right)^{T} \in \mathbb{R}^{3}$, more precisely $M_{\phi}=$ $\frac{d}{\sqrt{2}}\left(f_{3}+f_{4}-f_{1}-f_{2}\right), M_{\theta}=\frac{d}{\sqrt{2}}\left(f_{2}+f_{3}-f_{1}-f_{4}\right)$, and $M_{\psi}=\kappa_{1}\left(f_{2}+f_{4}-f_{1}-f_{3}\right)$, where $\kappa_{1}$ is a constant, therefore, by controlling $f_{i}$ unique and independent moments can be produced.

In outdoor conditions, gust winds are expected to involve drag side force and lift aerodynamic disturbances, related to the position dynamics, while the attitude suffers from the aerodynamic moments of rolling, pitching and yawing. For indoor conditions, flights are disturbed by gust winds generated through aerodynamic flows that circumvent the quadrotor, similar to the ground effect when an helicopter lands or a ceiling effect when a quadrotor establishes contact to the upper limit of a room. In addition, the gyroscopic moments of the quadrotor's rotors are present, regardless of the flying regimen [4]. Moreover, aerodynamic disturbances may render non-smooth behaviors, [11], which have been reported in association to some physical phenomena such as turbulence flows produced by multi-phase regimes in fluid dynamics, [12]. This particular class of disturbances are not necessarily Lipschitz and may include fractional-order terms, which are not everywhere differentiable, but with well-posed derivatives of some orders less than one, therefore, attitude disturbances $d_{a}(t)=\left(d_{\theta}(t), d_{\phi}(t), d_{\psi}(t)\right)^{T}$ are assumed continuous with fractional derivatives of some critical order less than one, [16].

\section{The Control Problem}

Noticing that the yaw angle $\psi$ and the altitude $z$ can be controlled independently, the problem is how to design $U \triangleq(\mathcal{T}, \tau)^{T}$ such that the stabilization of the underactuated translational axes $(x, y)$, subject to unknown uncertainties and disturbances is guaranteed. To this end, notice that if roll $\phi$ and pitch $\theta$ are designed to produce controlled displacement along $(x, y)$, while $\mathcal{T}$ controls the altitude $z$, then, the desired trajectories $\left(\theta_{d}, \phi_{d}\right)$ can be designed in such a way that a virtual position controller $\left(u_{x}, u_{y}\right)$ enforces convergence of $(x, y)$ to $\left(x_{d}, y_{d}\right)$. This is one way to resolve underactuation, which requires to solve the map between $\left(\theta_{d}, \phi_{d}\right)$ and $\left(u_{x}=\mathcal{T} r_{13}, u_{y}=\mathcal{T} r_{23}\right)$. However, this scheme needs very fast convergence of $(\psi, \theta, \phi) \rightarrow\left(\psi_{d}, \theta_{d}, \phi_{d}\right)$, with a wellposed attitude representation.

Thus, considering the properties of quaternions, [7], and the characteristics of fractional-order control, [16], it is proposed to solve the stated problem through a virtual position control that is achieved when the attitude tracking is exponentially assured, in addition with robustness to continuous but nondifferentiable disturbances and without chattering.

\section{Preliminaries on Fractional Calculus}

Fractional Calculus is based on differintegral operators of fractional-order, [13]. These operators possess inherent structural properties, which allow the modeling of nonlocal effects as well as the designing of control schemes with extended and improved features, [14], [15]. Consider the following differintegrals of order $\nu \in(0,1)$ :

- Riemann-Liouville fractional integral

$$
t_{0} I_{t}^{\nu} f(t)=\frac{1}{\Gamma(\nu)} \int_{t_{0}}^{t} \frac{f(\zeta)}{(t-\zeta)^{1-\nu}} d \zeta
$$

\section{- Extended Caputo fractional derivative}

$$
\begin{aligned}
{ }_{t_{0}}^{C} D_{t}^{\nu} f(t)= & \frac{f(t)-f\left(t_{0}\right)}{\Gamma(1-\nu)\left(t-t_{0}\right)^{\nu}}+ \\
& \frac{\nu}{\Gamma(1-\nu)} \int_{t_{0}}^{t} \frac{f(t)-f(\zeta)}{(t-\zeta)^{\nu+1}} d \zeta,
\end{aligned}
$$

The operator (5) was proposed in [16] to study the topological properties of regular enough continuous, but not necessarily 
integer-order differentiable, functions, and it is well-defined in the sense that complies to

${ }_{t_{0}}^{C} D_{t}^{\nu} t_{0} I_{t}^{\nu} f(t)=f(t)$ and $t_{0} I_{t}^{\nu}{ }_{t_{0}}^{C} D_{t}^{\nu} f(t)=f(t)-f\left(t_{0}\right)$,

for sufficiently good functions, [16]. In addition, operator (5) coincides with the conventional Caputo operator $\left({ }_{t_{0}}^{C} D_{t}^{\nu} f(t)={ }_{t_{0}} I_{t}^{\nu} \dot{f}(t)\right)$, [13], for differentiable functions.

\section{Attitude Control Design}

\section{A. A Singularity-free Kinematic Representation}

A rotation $\mathcal{R}: \mathcal{A} \rightarrow \mathcal{I}$ of a vector $\vec{v} \in \mathcal{A}$ can be parameterized by a unit quaternion $q \in \mathcal{S}(3)$. That is,

$\forall \vec{v} \in \mathcal{A} \exists q=\left(q_{0}, \vec{q}^{T}\right)^{T} \in \mathcal{S}^{3}$ such that $\mathcal{R} \vec{v}=q \otimes \vec{v} \otimes q^{*} \in \mathcal{I}$, where $q$ satisfies $q^{T} q=q_{0}^{2}+\vec{q}^{T} \vec{q}=1$, for scalar $q_{0}$ and $\vec{q} \in \mathbb{R}^{3}, q^{*}=\left(q_{0},-\vec{q}^{T}\right)^{T}$ is the conjugated of $q$. For two arbitrary quaternions $p$ and $q$ their quaternion product yields $p \otimes q=\left([p q]_{0}, p \odot q\right)^{T}$ resulting $[p q]_{0}=p_{0} q_{0}-\vec{p}^{T} \vec{q}$, and $p \odot q=p_{0} \vec{q}+q_{0} \vec{q}+\vec{p} \times \vec{q}$. Then, the rotation of an angle $\vartheta$ along the unit vector $\vec{\lambda}$ can be expressed in terms of $q=\cos (\vartheta / 2)+\vec{\lambda} \sin (\vartheta / 2)$. Hence, the orientation of a rigid body in $S O(3)$ with respect to $\mathcal{I}$ is expressed by a rotation $\mathcal{R}: \mathcal{A} \rightarrow \mathcal{I}$, resulting

$$
\mathcal{R}=I+2\left(\vec{q}^{\times}\right)^{2}+2 q_{0} \vec{q}^{\times} .
$$

The differential kinematics that maps angular velocities to quaternion rate is given by

$$
\dot{q}=\frac{1}{2}\left(\begin{array}{c}
-\vec{q}^{T} \\
{\left[q_{0} I+\vec{q}^{\times}\right]}
\end{array}\right) \omega
$$

which provides differential angle representation free of singularities, useful to design the attitude tracking controller.

\section{B. Design of the Error Manifold in Quaternions}

Inspired by [7], [17], let the orientation error manifold be

$$
S_{q}=\omega-\omega_{r}
$$

where $\omega_{r}$ is the nominal velocity reference given by

$$
\omega_{r}=\omega_{d}-\alpha \mathcal{R}_{d}^{T} \vec{q}_{e}+S_{d}
$$

where $q_{e}=q \otimes q_{d}^{*}=\left(q_{0 e}, \vec{q}_{e}^{T}\right)^{T}$ is the attitude quaternion tracking error, $\alpha>0$, and $\mathcal{R}_{d}$ represents the desired rotation matrix parameterized in terms of the desired unit quaternion $q_{d}$, and $S_{d}=S\left(t_{0}\right) e^{-\kappa\left(t-t_{0}\right)}$, with $\kappa>0$. Substituting (9) into (8), the latter becomes

$$
\begin{aligned}
S_{q} & =S-S_{d}, & & \text { sliding surface } \\
S & =\omega_{e}+\alpha \mathcal{R}_{d}^{T} \vec{q}_{e}, & & \text { nonlinear error manifold }
\end{aligned}
$$

where $\omega_{e}=\omega-\omega_{d}$, for $\omega_{d}$ the desired angular velocity. Notice that $S_{q}\left(t_{0}\right)=0$ for any initial condition and (6) suggests that $\mathcal{R}_{d}=I+2\left(\vec{q}_{d}^{\times}\right)^{2}+2 q_{0 d} \vec{q}_{d}^{\times}$. Moreover, from (7), the desired quaternion rate can be written as

$$
\dot{q}_{d}=\frac{1}{2}\left(\begin{array}{c}
-\vec{q}_{d}^{T} \\
{\left[q_{0 d} I+\vec{q}_{d}^{\times}\right]}
\end{array}\right) \omega_{d}
$$

such that,

$$
\dot{q}_{e}=\frac{1}{2}\left(\begin{array}{c}
-\vec{q}_{e}^{T} \\
{\left[q_{0 e} I+\vec{q}_{e}^{\times}\right]}
\end{array}\right) \mathcal{R}_{d} \omega_{e} .
$$

The motivation to design this quarternion-based error manifold is now clear: if a controller ensures that $S_{q}=0$ for some finite time, then $\omega_{e}=-\alpha \mathcal{R}_{d}^{T} \vec{q}_{e}+S_{d}$. Since $S_{d}$ vanishes exponentially fast independently of any state of the system, assume $S \approx 0$ at time $t_{s}$, then,

$$
\dot{q}_{0 e}=\frac{\alpha}{2}\left(1-q_{0 e}^{2}\right) \quad \text { and } \quad \dot{\vec{q}}_{e}=-\frac{\alpha}{2} q_{0 e} \vec{q}_{e},
$$

whose solution is exponential without singularities of representation, leading to exponential attitude tracking, [18].

\section{Open-loop Error Dynamics}

Aiming at designing a controller that enforces $S_{q}=0$ as a sliding surface in finite-time, let the open-loop error dynamics be, by adding $-\mathbf{J} \dot{\omega}_{r}$ to (3),

$$
\mathbf{J} \dot{S}_{q}=\tau-\mathbf{J} \dot{\omega}_{r}-\omega^{\times} \mathbf{J} \omega+d_{a}(t)
$$

Assuming $\mathbf{J}$ is uncertain, (14) becomes

$$
\mathbf{J} \dot{S}_{q}=\tau+\varphi(t)
$$

for $\varphi(t)=-\mathbf{J} \dot{\omega}_{r}-\omega^{\times} \mathbf{J} \omega+d_{a}(t)$. It is clear that the topological properties of $\varphi(t)$ are intrinsically related with those of $d_{a}$. At this point, the control problem is to design a continuous chatter-less controller $\tau$ such that $q \rightarrow q_{d}, \omega \rightarrow \omega_{d}$ for a continuous but not necessarily differentiable disturbance $\varphi(t)$, which is solved if $\tau$ stabilizes $S_{q}=0$ in finite-time.

\section{Design of the Fractional Attitude Controller}

Assume that, for some $\varsigma \in(0,1)$, the derivatives of every order $\nu \in(0, \varsigma)$ of $d_{a}(t)$ are bounded on any compact domain $\Omega \subset \mathbb{R}$, then $\sup _{(a, b) \subset \Omega}\left\|\left.\right|_{a} ^{C} D_{t}^{\nu} \varphi(t)\right\|_{t=b} \in \mathbb{R} \forall \nu \in(0, \varsigma)$, and, when a given maximum $\varsigma_{\max }$ satisfies the above conditions it is known as the critical order of $\varphi(t)$, [16].

Now, consider the following model-free fractional control

$$
\tau(t)=-k_{t_{0}} I_{t}^{\nu} \operatorname{sign}\left(S_{q}(t)\right)
$$

for $\nu<\varsigma_{\max }$, and $k$ a feedback gain. Substituting (16) into (15), the term-wise closed-loop attitude dynamics for the $i$-th component of $S_{q}$ is

$$
J_{i i} \dot{S}_{q i}=J_{i i} \dot{S}_{q i}\left(t_{n}\right)-k_{t_{n}} I_{t}^{\nu} \operatorname{sign}\left(S_{q i}\right)+{ }_{t_{n}} I_{t}^{\nu}{ }_{a}^{C} D_{t}^{\nu} \varphi_{i}^{\prime}
$$

where $J_{i j}$ is the $i j$-entry of $\mathbf{J}, \varphi_{i}^{\prime}(t)=-\mathbf{J} \dot{\omega}_{r}-\omega^{\times} \mathbf{J} \omega+d_{a}(t)+$ $\sum_{j \neq i} J_{i j} S_{q j}+\varrho_{i}(t)$, and the term $\varrho_{i}(t)$ is the memory effect before $t_{n}$, which affects the system behavior for any $t \geq t_{n}$. This term is casted as an endogenous disturbance to allows the application of the resetting memory principle proposed in [19]. Such principle consists in reestablishing the lower terminal at each time instant when $S_{q i}(t)$ crosses the origin, that is, when $S_{q i}\left(t_{n}\right)=0$, obtaining the monotonously increasing sequence of nonnegative real numbers $\left\langle t_{n}\right\rangle_{n \in \mathbb{N}}$. Thus, consider the following Theorem.

Theorem 1: Consider the closed-loop equation (17), with $\nu \in(0,1)$, and let $c=\sup \left|{ }_{a}^{C} D_{t}^{\nu} \varphi_{i}^{\prime}(t)\right| \in \mathbb{R}$. Then, for 
$k>\frac{3+\nu}{1-\nu} c$, there exists a finite time $t_{s} \in \mathbb{R}$ such that $\left(S_{q i}(t), \dot{S}_{q i}(t)\right)=(0,0) \forall t \geq t_{s}$.

Proof: Proof is similar to that in Theorem 1 of [19]. However, in contrast to [19], the fractional integral is not reset for computing the control signal. This demonstrates that

$$
\left|\dot{S}_{q i}\left(t_{n}\right)\right| \leq \mu^{n}\left|\dot{S}_{q i}\left(t_{0}\right)\right|, \forall n \in \mathbb{N},
$$

for $\mu=\frac{k+c}{k-c}(1+\nu)-1<1$, then, $\dot{S}_{q i}\left(t_{n}\right) \rightarrow 0$. The time instant $t_{s}$, such that $\left(S_{q}(t), \dot{S}_{q}(t)\right)=(0,0) \forall t \geq t_{s}$, is given by the geometric series $t_{s}=t_{0}+\sum_{n=0}^{\infty}\left(t_{n+1}-t_{n}\right)$, thereby, $t_{s} \leq t_{0}+\left[\frac{J_{i i}\left|\dot{S}_{q i}\left(t_{0}\right)\right| \Gamma(\nu+2)}{k-c}\right]^{1 / \nu} \frac{1}{1-\mu^{1 / \nu}} \in \mathbb{R}$.

\section{E. Solving Underactuation: Desired Attitude design}

Theorem 1 establishes that $q_{e} \rightarrow 0$ at $t \geq t_{s}$, and consequently, $(\psi, \theta, \phi)^{T} \rightarrow\left(\psi_{d}, \theta_{d}, \phi_{d}\right)^{T}$ exponentially. Thus, consider the desired position control be $u=\mathcal{T} \mathcal{R}_{d} e_{z}$, whose magnitude is $\mathcal{T}>0$ (since it sustain the quadrotor on the air) with a direction $\mathcal{R}_{d} e_{z}$. Since $\|u(t)\|=\|\mathcal{T}\|\left\|r_{d 3}\right\|=\|\mathcal{T}\|$, where $r_{d 3}=\mathcal{R}_{d} e_{z}$ is the third column of the desired rotation matrix, one has that $r_{d 3}=u(t) / \mathcal{T}$, which leads to

$$
\begin{aligned}
\phi_{d} & =\arcsin \left(\frac{u_{1} \sin \psi_{d}-u_{2} \cos \psi_{d}}{\mathcal{T}}\right) \\
\theta_{d} & =\arctan \left(\frac{u_{1} \cos \psi_{d}+u_{2} \sin \psi_{d}}{u_{3}}\right),
\end{aligned}
$$

depending solely on $u=\left(u_{1}, u_{2}, u_{3}\right)^{T}$ and $\psi_{d}$, such that, $u_{3}>0$ compensates gravity effects. The solution of the kinodynamic constraint (2) gives rise to a well-posed desired angular velocity $\omega_{d}=\left(\omega_{1 d}, \omega_{2 d}, \omega_{3 d}\right)^{T}$, producing

$$
\begin{aligned}
\omega_{1 d} & =\left(\mathcal{T} \gamma_{d}-\delta_{d} \dot{\mathcal{T}}\right) / \mathcal{T} h_{0}-\dot{\psi}_{d} \beta_{d} / h_{1} \\
\omega_{2 d} & =\left(\alpha_{d} u_{3}-\dot{u}_{3} \beta_{d}\right) h_{0} / \mathcal{T} h_{1}^{2}+\dot{\psi}_{d} \delta_{d} u_{3}^{2} / \mathcal{T} h_{1} \\
\omega_{3 d} & =\dot{\psi}_{d} u_{3}^{2} h_{0} / \mathcal{T} h_{1}-\left(\alpha_{d} u_{3}-\dot{u}_{3} \beta_{d}\right) \delta_{d} / \mathcal{T} h_{1}^{2}
\end{aligned}
$$

where $h_{0}^{2}=\mathcal{T}^{2}-\delta_{d}^{2}>0$ and $h_{1}^{2}=\left(\beta_{d}^{2}+u_{3}^{2}\right)>0$, for

$$
\begin{aligned}
\alpha_{d} & =\dot{u}_{1} \cos \psi_{d}-u_{1} \dot{\psi}_{d} \sin \psi_{d}+\dot{u}_{2} \sin \psi_{d}+u_{2} \dot{\psi}_{d} \cos \psi_{d} \\
\beta_{d} & =u_{1} \cos \psi_{d}+u_{2} \sin \psi_{d} \\
\gamma_{d} & =\dot{u}_{1} \sin \psi_{d}+u_{1} \dot{\psi}_{d} \cos \psi_{d}-\dot{u}_{2} \cos \psi_{d}+u_{2} \dot{\psi}_{d} \sin \psi_{d} \\
\delta_{d} & =u_{1} \sin \psi_{d}-u_{2} \cos \psi_{d} \\
\dot{\mathcal{T}} & =\left(u_{1} \dot{u}_{1}+u_{2} \dot{u}_{2}+u_{3} \dot{u}_{3}\right) / \mathcal{T}
\end{aligned}
$$

Note that, when the desired angular velocity is computed to resolve underactuation, the time derivative of $u$ is required, which can be computed following a similar procedure as that proposed in [18].

\section{Position Control Design}

By considering the virtual control $u=\mathcal{T} \mathcal{R} e_{z}$, system (1) can be written as

$$
m \dot{S}_{p}=-u+\varphi_{p}
$$

where $\varphi_{p}=m g e_{z}-m \ddot{\xi}_{r}+d_{p}(t)$ includes endogenous and exogenous effects, for

$$
\begin{array}{rlrl}
S_{p} & =\dot{\xi}-\dot{\xi}_{r} & \underline{\text { extended error manifold }} & \text { (24) } \\
\dot{\xi}_{r} & =\dot{\xi}_{d}-\alpha_{p} \xi_{e}+\bar{S}_{d}-\gamma \sigma & \text { nominal reference } & \text { (25) } \\
\dot{\sigma} & =\tanh \left(\lambda_{p} S_{\xi}\right) &
\end{array}
$$

with $\lambda_{p}, \alpha_{p}$ and $\gamma$ positive definite diagonal matrices, $\xi_{d}$ is the smooth desired position, $\xi_{e}=\xi-\xi_{d}$ stands for the tracking error and $\bar{S}_{d}=S_{\xi}\left(t_{0}\right) e^{-\bar{\kappa}\left(t-t_{0}\right)}$ for $\bar{\kappa}>0$. Notice that, for large enough elements of $\lambda_{p}$, it can be considered that $\tanh \left(\lambda_{p} S_{\xi}\right) \approx \operatorname{sign}\left(S_{\xi}\right)$. Equations (25)-(26) allow equation (24) to be rewritten as

$$
\begin{array}{llc}
S_{p} & =S_{\xi}-\bar{S}_{d} & \text { sliding surface } \\
S_{\xi} & =\dot{\xi}_{e}+\alpha_{p} \xi_{e} & \text { linear error manifold }
\end{array}
$$

The quasi-continuous sliding motion condition is demonstrated in the following Theorem.

Theorem 2: Consider the position dynamics (1) in closedloop with

$$
u=K_{d} S_{p}
$$

Then, robust exponential convergence to a small vicinity of the origin is assured for large enough elements of the positive definite feedback matrices $\gamma$ and $K_{d}$.

Proof: The proof follows similar arguments of [17] and [18], however, essentially it is shown that a quasi-sliding mode is established at $\left\|S_{\xi}\right\| \leq c_{0}$, where $c_{0}$ can be made arbitrarily small by tuning $\lambda_{p}$ and $K_{d}$.

\section{ANAlysis BASED On Simulations}

A comparison analysis based on simulations is carried out to highlight the advantages of the fractional control.

The Simulator. Two simulations, for $\nu=0$ and $\nu=0.5$, are considered. It is important to note that $\nu=0$ corresponds to the classical sliding mode control. Simulations run on Simulink in Matlab 2013a, based on the Euler integrator with a sampling rate of $10 \mathrm{KHz}$, and the CRONE method to compute differintegrals, with a transfer function of 100-th order on $[1 \mathrm{mHz}, 1 \mathrm{KHz}]$.

The plant and the task. Consider an UAV quadrotor with $m=0.442$, and $\mathbf{J} \approx \operatorname{diag}\{0.002,0.002,0.004\} \mathrm{kgm}^{2}$. The task is $x_{d}=-0.5+0.5 \cos (0.2 t), y_{d}=0.5 \sin (0.2 t), z_{d}=$ $0.3+0.4 \sin (0.2 t)$, and $\psi_{d}=0.1 \sin (0.1 t)$.

Control Parameters. For all cases consider the position control parameters $K_{d}=20 I_{3 \times 3}, \gamma=0.1 I_{3 \times 3}, \lambda_{p}=10 I_{3 \times 3}$, $\alpha_{p}=0.2 I_{3 \times 3}$, and $\bar{\kappa}=1$. The attitude control gains are $\kappa=5$, $\alpha=20, c=0.7, k=c(3+\nu) /(1-\nu)$, for both $\nu=0$ and 0.5 . Consider $d_{a}(t)=[\sin (t), \sin (t+2 \pi / 3), \sin (t+4 \pi / 3)]^{T}+$ $(1,1,1)^{T}{ }_{0} I_{t}^{0.6} \operatorname{random}_{(0,20)}(t)$ for the attitude disturbance, see Figure 2, with a random function of mean equal 0 and variance of 20 .

Results. For both cases, $\nu=0$ and $\nu=0.5$, almost the same position performance is obtained since the (virtual) position control results from integrating two times the attitude control, then, high frequency effects of $\tau$ in the position dynamics cannot be clearly appreciated. Thus, only attitude control and sliding surfaces signals are shown.

Fig. 3 highlights that the system performance is improved as the order of integration increases. However, a large value of $\nu$ implies a lack of robustness wrt more aggressive disturbances. Then, the tuning of $\nu$ allows to design preciser actions of control by considering further properties neglected in the integer-order case. Note that exact rejection is achieved just in the fractional-order case. 


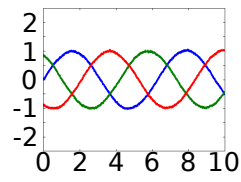

Fig. 2. Continuous but non-differentiable disturbance: $t$ vs $d_{a}(t)$.

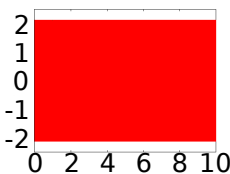

(a) $\nu=0: t$ vs $\tau$

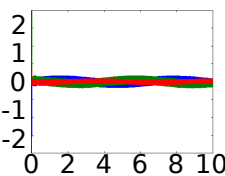

(c) $\nu=0: t$ vs $S_{q}$

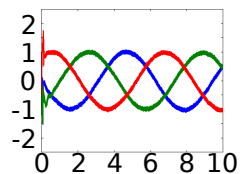

(b) $\nu=0.5: t$ vs $\tau$

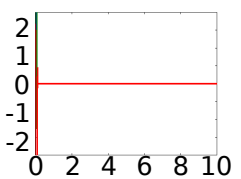

(d) $\nu=0.5: t$ vs $S_{q}$
Fig. 3. Numerical results based on simulations

\section{EXPERIMENTAL STUDY}

Experimental tests were developed in the Heudiasyc Laboratory at the Université de Technologie de Compiègne. The equipment is composed of a motion capture system Optitrack that provides position and $\psi$ in the quadrotor frame, and a Parrot AR Drone 2.0 quadrorotor with custom made programming platform onto two personal computers, one to process data from the Optitrack and other to handle bilateral communication to the quadrotor. An SDK provided by Parrot allows the quadrotor to be controlled. However, it is not possible to directly implement our controllers since the quadrotor has closed architecture. We solved this problem through the autopilot Paparazzi developed by teams from TU Delft university, [23]. In this platform, the communication protocols between the onboard main processor and the peripheral devices have been decoded, and by using these protocols and our own software framework, the proposed controller can be directly implemented. The controller is embedded on the quadrotor processing system using Gumstix architecture that handles additionally all onboard processing and interruptions of comms, sensors and actuators, while the Optitrack PC send $\psi, x, y, z$ DoFs, and $\phi$ and $\theta$ are obtained reading an IMU. The time base runs at $200 \mathrm{~Hz}$, and the whole programming is coded in $\mathrm{C}++$ within Linux Mint $17 \mathrm{KDE}$ environment using CodeBlocks 10. The Grünwald-Letnikov method [13] is programmed to compute numerical differintegrals with a maximum of 5000 memory elements in each iteration. Figure 4 shows the main elements of the control architecture. As it is customary, the control input is saturated to protect the integrity of the equipment, corresponding to the normalized sum of the desired control signals less than or equal to 1 . Roughly speaking, the virtual position control is computed on the onboard processor of the quadrotor, which receives the translational position and velocity from the Optitrack via Wifi. Once the position control is calculated, it passes through the mapping generating the desired quaternion and angular velocities. Using this information with data from the IMU, the attitude control is calculated. Finally, the output from the attitude control is mapped to the corresponding desired angular velocities of the propellers ( $w_{p_{i}}$ for $i \in\{1,2,3,4\}$ ), which are handled by the rotors' drivers, see in Figure 5 .

\section{A. Experimental Conditions}

Initial conditions are $z\left(t_{0}\right)=0.75 \mathrm{~m}$, and desired operational trajectory stands for the circle $\left[x_{d}, y_{d}\right]=[0.5 \sin (0.2 t)+$ offset, $0.5 \cos (0.2 t)+$ offset $] \mathrm{m}$ while $z_{d}=0.4 \sin (0.2 t)+0.75$, and a yaw ramp $\psi_{d}=15^{\circ} t$. The offset is tuned to comply with small error on initial conditions, under a trial-and-error basis. Attitude and position control gains are $\nu=0.6, \kappa=1, \alpha=$ $9, k=0.8, K_{d}=0.35 I_{3 \times 3}, \alpha_{p}=4 I_{3 \times 3}, \lambda_{p}=10 I_{3 \times 3}$, $\gamma=0.15 I_{3 \times 3}, \bar{\kappa}=5$.

\section{B. Experimental Results}

Desired positions are quite demanding to exhibit the strong couplings of the nonlinear dynamics to test the control capabilities. In Fig. 6, position tracking is presented where the apparent lag is due to the multi-sampling and latency of multiple processes implemented to run the experiments, as well as sensor noise and quantization, typical of this sort of experiments. The same Fig. 6 shows the continuous control signals handled by the rotors' drivers in a normal operational regime. Fig. 7 shows $\psi$ tracking the ramp reference, and the virtual position control, which is certainly smooth for such large $\lambda_{p}=10 I_{3 \times 3}$.

\section{CONCLUSIONS}

A model-free position tracking control for quadrotors is presented based on the virtual control concept. In order to succeed in position tracking, the attitude control induces a fractional sliding mode that rejects not necessarily differentiable disturbances, inducing exponentially the virtual position controller.

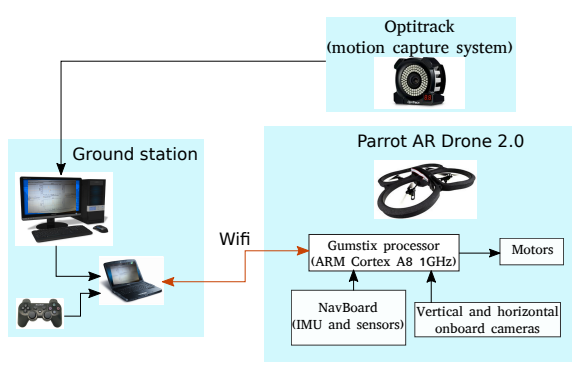

Fig. 4. Control architecture of the experimental platform

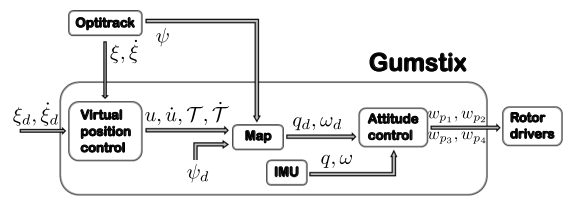

Fig. 5. Scheme of the control algorithm 


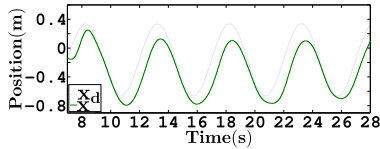

(a) $x$ vs $t$

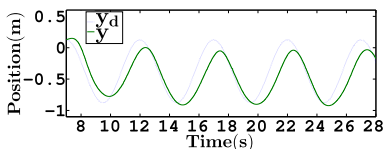

(c) $y$ vs $t$

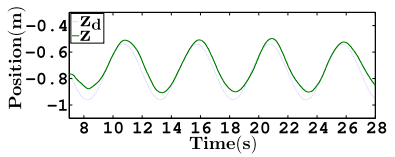

(e) $z$ vs $t$

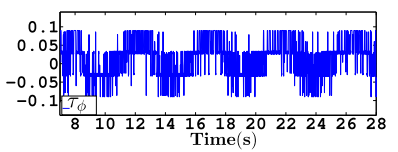

(b) $\tau_{\phi}$ vs $t$

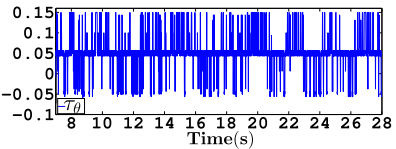

(d) $\tau_{\theta}$ vs $t$

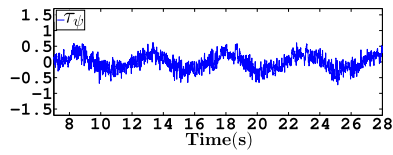

(f) $\tau_{\psi}$ vs $t$
Fig. 6. Experiments. Position tracking (left) and control signals (right).

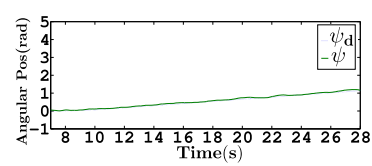

(a) $\psi$ vs $t$

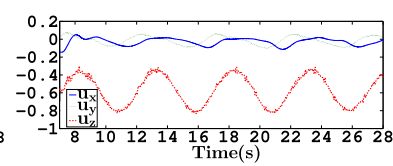

(b) $u$ vs $t$
Fig. 7. Experiments. Yaw tracking $\psi$ and virtual position controller $u$.

A comparison analysis based on simulations highlights the advantages of the fractional-order control actions, and rigorous experimental developments are tested in a soft real-time platform. Future work includes to enhance the performance of the attitude controller with a hard real-time patch based on Xenomai, as well as to increase the onboard processing for more endurance testing.

\section{ACKNOWLEDGEMENTS}

Authors acknowledge partial support from Conacyt-Mexico under Basic Research Grants 133346, and 133544, as well as the programm Investissements d'avenir programme through the Robotex Equipment of Excellence Grant ANR-10-EQPX44 of France, and the Labex MS2T Framework of France through the program "Investments for the future" Grant ANR11-IDEX-0004-02 of the National Agency for Research.

\section{REFERENCES}

[1] D. Cabecinhas, R. Cunha and C. Silvestre, "A Globally Stabilizing Path Following Controller for Rotorcraft With Wind Disturbance Rejection," IEEE Transactions on Control Systems Technology, vol. 23, no. 2, pp. 708-714, 2015.

[2] L. Wang and J. Su, "Robust Disturbance Rejection Control for Attitude Tracking of an Aircraft," IEEE Transactions on Control Systems Technology, vol. 23, no. 6, pp. 2361-2368, 2015.

[3] Madani, T., Benallegue, A. "Backstepping Control for a Quadrotor Helicopter," Proc. of IEEE/RSJ International Conference on in Intelligent Robots and Systems, 3255-3260, 2006.

[4] H. Ramirez-Rodriguez, V. Parra-Vega, A. Sanchez-Orta and O. GarciaSalazar, "Robust Backstepping Control Based on Integral Sliding Modes for Tracking of Quadrotors," Journal of Intelligent Robotic Systems, vol. 73, no. 4, pp. 51-66, 2014.

[5] T. Lee, M. Leoky, and N. H. McClamroch, "Geometric Tracking Control of a Quadrotor UAV on SE (3)," Proc. of IEEE Conference on Decision and Control, pp. 5420-5425, 2010.
[6] M. Bergamasco and M. Lovera, "Identification of Linear Models for the Dynamics of a Hovering Quadrotor," IEEE Transactions on Control Systems Technology, vol. 22, no. 5, pp. 1696-1707, 2014.

[7] V. Parra-Vega, A. Sanchez, C. Izaguirre, O. Garcia, F. Ruiz-Sanchez, "Toward Aerial Grasping and Manipulation with Multiple UAVs," Journal of Intelligent Robotic Systems, vol. 70, no. 4, pp. 575-593, 2013.

[8] A.J. Muñoz-Vázquez, V. Parra-Vega, A. Sáncez-Orta, O. García and C. Izaguirre-Espinosa, "Attitude Tracking Control of a Quadrotor based on Absolutely Continuous Fractional Integral Sliding Modes," In Proc. of The IEEE Conference on Control Applications, pp. 717-722, 2014.

[9] A. Tayebi and S. McGilvray, "Attitude Stabilization of a VTOL Quadrotor Aircraft," IEEE Transactions on Control Systems Technology, vol. 14, no. 3, pp. 562-571, 2006.

[10] B. Etkin, and L. D. Reid, Dynamics of Flight, New York: Wiley, 1959.

[11] J. Humphrey, C. Schuler and B. Rubinsky, "On the Use of the Weierstrass-Mandelbrot Function to Describe the Fractal Component of Turbulent Velocity," Fluid Dynamics Research, vol. 9, 81-95, 1992.

[12] N. I. Kolev, Multiphase Flow Dynamics 2: Thermal and Mechanical Interactions, Berlin Heidelberg: Springer, 2007.

[13] Podlubny, I. (1999). Fractional Differential Equations, San Diego CA: Academic Press, 1999.

[14] H. Li, Y. Luo and Y.Q. Chen, "A Fractional Order Proportional and Derivative (FOPD) Motion Controller: Tuning Rule and Experiments," IEEE Transactions on Control Systems Technology, vol. 18, no. 2, pp. 516-520, 2010.

[15] Y. Luo, Y.Q. Chen, H.S. Ahn and Y. G. Pi, "Fractional Order Periodic Adaptive Learning Compensation for State-dependent Periodic Disturbance," IEEE Transactions on Control Systems Technology, vol. 20, no. 2, pp. 465-472, 2012.

[16] A.J. Muñoz-Vázquez, V. Parra-Vega and A. Sánchez-Orta, "Uniformly Continuous Differintegral Sliding Mode Control of Nonlinear Systems subject to Hölder Disturbances," Automatica, vol. 66, pp. 179-184, 2016.

[17] V. Parra-Vega, S. Arimoto, Y. Liu G. Hirzinger and P. Akella, "Dynamic Sliding PID Control for Tracking of Robot Manipulators: Theory and Experiments," IEEE Transactions on Robotics and Automation, vol. 19, no. 6, pp. 967-976, 2003.

[18] A. Sanchez-Orta, V. Parra-Vega, C. Izaguirre-Espinosa and O. Garcia, "Position-Yaw Tracking of Quadrotors," Journal of Dynamic Systems, Measurement, and Control, vol. 137, no. 6, pp. 061011, 2015.

[19] C. Izaguirre-Espinosa, A.J. Muñoz-Vázquez, A. Sánchez-Orta, V. ParraVega and P. Castillo "Attitude Control of Quadrotors based on Fractional Sliding Modes: Theory and Experiments," IET Control Theory \& Applications, vol. 10, no. 7, pp. 825-832, 2016.

[20] A. Oustaloup, B. Mathieu and P. Lanusse, "The CRONE Control of Resonant Plants: Application to A Flexible Transmission," European J. Control, vol. 1, pp. 113-121, 1995.

[21] M. Önder-Efe, "Integral Sliding Mode Control of a Quadrotor with Fractional Order Reaching Dynamics," Transactions of the Institute of Measurement and Control, vol. 33, no. 8, pp. 985-1003, 2011.

[22] J. L Han, L. Di, C. Coopmans and Y.Q. Chen, "Pitch Loop Control of a VTOL UAV using Fractional Order Controller, Journal of Intelligent \& Robotic Systems, vol. 73, no. 1, pp. 187-195, 2014.

[23] http://wiki.paparazziuav.org/wiki/AR_Drone_2/getting_started. 